\title{
AN ALTERNATIVE LAPLACE DISTRIBUTION
}

\author{
C. SATHEESH KUMAR ${ }^{\star}$ \\ Department of Statistics, University of Kerala, Trivandrum, Kerala, India \\ Email: drcsatheeshkumar@gmail.com \\ Rosmi Jose \\ Department of Statistics, University of Kerala, Trivandrum, Kerala, India \\ Email: rosmijose19@gmail.com
}

\begin{abstract}
SUMMARY
In this paper, we propose an alternative version to the Laplace distribution which we named as "alternative Laplace distribution (ALD)" and discuss some of its important properties. A location-scale extension of the ALD is considered and the maximum likelihood estimation procedures for estimating its parameters is described. Further, the distribution is fitted to certain real life data sets for illustrating the utility of the model. A simulation study is carried out to examine the performance of likelihood estimators of the parameters of the distribution.

Keywords and phrases: Laplace distribution, Maximum likelihood estimation, Model selection, Order statistics, Reliability measures, Rényi entropy, Simulation.
\end{abstract}

\section{Introduction}

The Laplace distribution, also called the double exponential distribution was considered as the first continuous distribution of unbounded support in statistical literature and it is named after the French mathematician Pierre-Simon Laplace. The Laplace distribution is the distribution of the difference of two independent random variables with identical exponential distributions. It is often used to model phenomena with heavy tails or when data has a higher peak than the normal distribution. The Laplace distribution is defined through the probability density function (p.d.f)

$$
f_{1}(x ; \mu, \sigma)=\frac{1}{2 \sigma} e^{-\frac{|x-\mu|}{\sigma}}
$$

for $x \in R=(-\infty, \infty), \mu \in R$ and $\sigma>0$. The Laplace distribution with location parameter zero and scale parameter one is called the classical Laplace distribution and its p.d.f is given by

$$
f_{2}(x)=\frac{1}{2} e^{-|x|}
$$

for $x \in R$.

\footnotetext{
* Corresponding author

(C) Institute of Statistical Research and Training (ISRT), University of Dhaka, Dhaka 1000, Bangladesh.
} 
Several modifications of the Laplace distribution are currently available in the literature. Some recent studies in this respect were made by Cordeiro and Lemonte (2011), Jose and Thomas (2014), Liu and Kozubowski (2015), Mahmoudvand et al. (2015), Kozubowski et al. (2016), Nassar (2016) and $\mathrm{Li}$ (2017). Laplace distribution has wide range of applications in real life to model and analyze data sets in engineering, financial, industrial, environmental and biological fields.

In this paper, we consider a generalization of the classical Laplace distribution and we call it as "the alternative Laplace distribution"(or in short "ALD"). As compared to the Laplace distribution, the main advantage of the ALD is that it possesses both unimodal and bimodal shapes. Also, ALD accommodates relatively more variation in peakedness compared to Laplace distribution and consequently it also possess heavier tails than the Laplace distribution. Due to these reasons, ALD serves better than the Laplace distribution and can be used to model several real life situations. Through this paper, we also consider a location-scale extension of this distribution which we termed as the "extended alternative Laplace distribution (EALD)" and attempt to highlight the relevance of the EALD, compared to the existing Laplace distribution with the help of certain real life data sets.

The rest of the paper is organized as follows: In section 2, we present the definition of the ALD and obtain some basic properties of the distribution through deriving expressions for its c.d.f, moments, skewness, kurtosis, mean deviation about mean, moment generating function, characteristic function and cumulant generating function. A location-scale extension of the ALD is discussed in this section as a remark. Certain reliability measures of the ALD are given in section 3 and in section 4 we discuss some order statistics related properties of this distribution. Section 5 contains the derivation of the Rényi entropy measure of the ALD. Section 6 deals with the maximum likelihood estimation of ALD and EALD and in section 7 we illustrate the estimation procedures with the help of certain real life data sets. A simulation study has been carried out in section 8 for examining the performance of likelihood estimators for the parameters of the EALD. Finally, we conclude the findings of the study through section 9 .

\section{Definition and Properties}

In this section, first we present the definition of the ALD and investigate some of its important properties.

Definition 2.1. A random variable $X$ is said to follow alternative Laplace distribution (ALD) with parameter $\alpha$ if its p.d.f is given by

$$
f(x ; \alpha)=\frac{1}{2(\alpha+1)}(1+\alpha|x|) e^{-|x|},
$$

for $x \in R$ and $\alpha \geq 0$.

A distribution with p.d.f (2.1) hereafter we denoted as $\operatorname{ALD}(\alpha)$. Clearly $\operatorname{ALD}(0)$ is the classical Laplace distribution with p.d.f (1.2). Probability plots of the $\operatorname{ALD}(\alpha)$ for $\alpha \leq 1$ are presented in Figure 1 and that of $\alpha>1$ are given in Figure 2.

We obtain the c.d.f, mode and expression for $r^{t h}$ raw moment of the ALD through the following prepositions. We omit their proofs, since they are straightforward. 


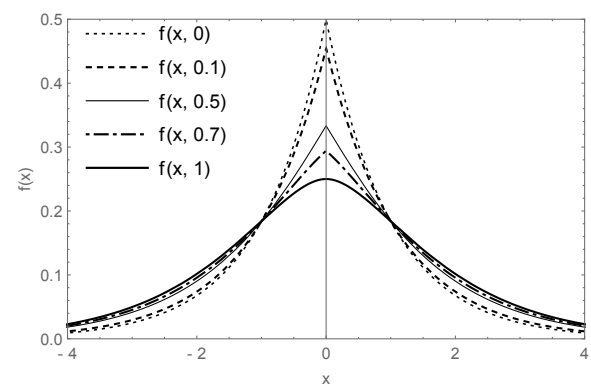

Figure 1: The probability density function of the ALD for $\alpha \leq 1$

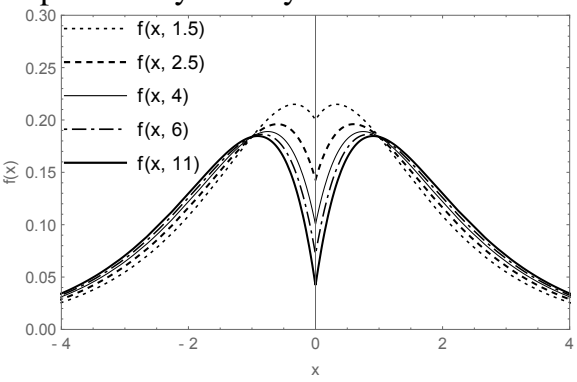

Figure 2: The probability density function of the ALD for $\alpha>1$

Figure 3: The probability density function of the ALD for selected values of $\alpha$

Proposition 2.1. The cumulative distribution function (c.d.f) of the ALD with p.d.f (2.1) is:

$$
F(x ; \alpha)=\left\{\begin{array}{cc}
\frac{(1+\alpha-\alpha x) \mathrm{e}^{x}}{2(\alpha+1)}, & x<0 \\
1-\frac{(1+\alpha+\alpha x) \mathrm{e}^{-x}}{2(\alpha+1)}, & x \geq 0
\end{array}\right.
$$

Figure 4 represents the plots of the cumulative distribution function of the ALD for various parameter values.

Proposition 2.2. The mode of the ALD is given by

$$
\text { Mode }=\left\{\begin{array}{cc}
\frac{\alpha-1}{\alpha} \text { and } \frac{1-\alpha}{\alpha}, & \text { if } \alpha>1 \\
0, & \text { otherwise }
\end{array}\right.
$$

Proposition 2.3. For $r>0$, the $r^{\text {th }}$ raw moment, $\mu_{r}^{\prime}$ of the ALD is

$$
\mu_{r}^{\prime}=E\left(X^{r}\right)=\left\{\begin{array}{cl}
\frac{r !(1+\alpha(r+1))}{\alpha+1}, & \text { if } \mathrm{r} \text { is even } \\
0, & \text { if } \mathrm{r} \text { is odd }
\end{array}\right.
$$

As a consequence of proposition 2.3, we have the following corollary. 


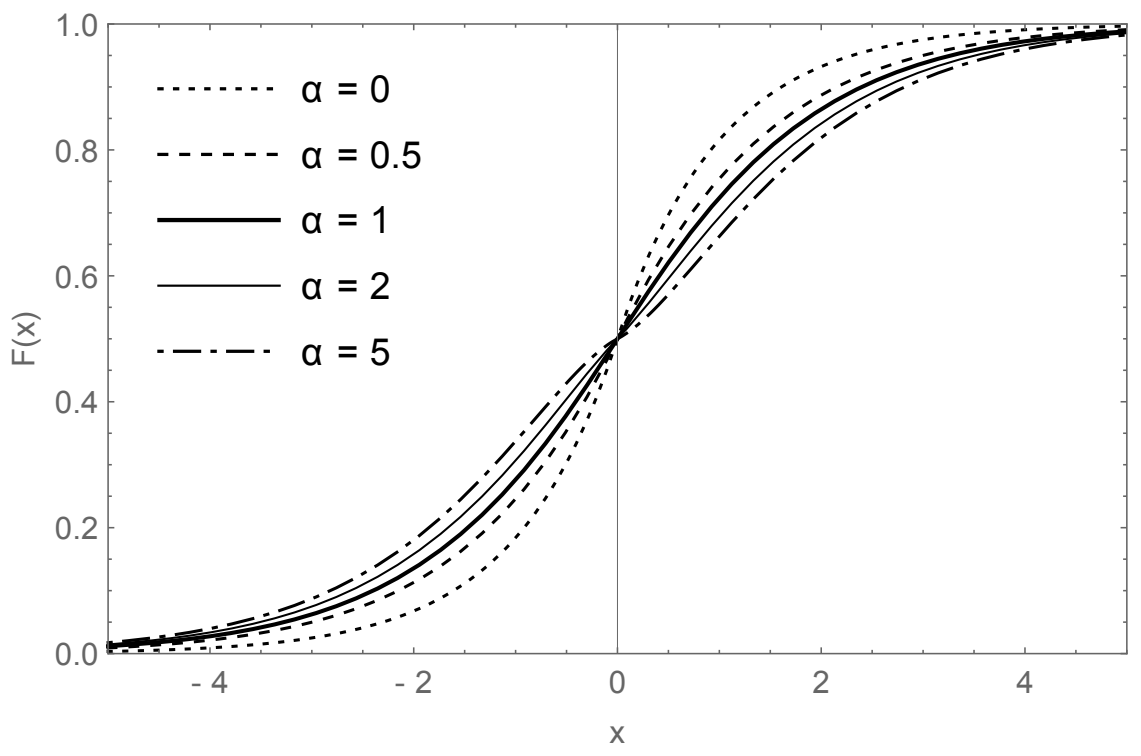

Figure 4: The cumulative distribution function of the ALD for different values of $\alpha$

Corollary 2.1. The coefficient of skewness $\left(\beta_{1}\right)$ and the coefficient of kurtosis $\left(\beta_{2}\right)$ of the ALD are respectively,

$$
\beta_{1}=\frac{\mu_{3}^{2}}{\mu_{2}^{3}}=0
$$

and

$$
\beta_{2}=\frac{\mu_{4}}{\mu_{2}^{2}}=\frac{3 !(5 \alpha+1)(\alpha+1)}{(3 \alpha+1)^{2}} .
$$

Proof follows from the $2^{\text {nd }}, 3^{\text {rd }}$ and $4^{\text {th }}$ central moments $\left(\mu_{2}, \mu_{3}\right.$ and $\left.\mu_{4}\right)$ of the ALD as $\mu_{2}=$ Variance $=\frac{2 !(3 \alpha+1)}{\alpha+1}, \mu_{3}=0$ and $\mu_{4}=\frac{4 !(5 \alpha+1)}{\alpha+1}$. These expressions can be easily derived from (2.4).

From Corollary 2.1, it is quiet trivial that the ALD is symmetric and leptokurtic in nature. It can be verified that $\beta_{2}$ ranges from 3.33 to 6 for the ALD whereas Laplace distribution has a constant kurtosis 6 . Therefore, ALD can accommodate data sets with variation in peakedness than the Laplace distribution.

Next we derive an expression for the $r^{t h}$ absolute moment of the ALD through the following proposition.

Proposition 2.4. For $r \geq 1$, the $r^{t h}$ absolute moment $\delta_{r}$ of the ALD is given by

$$
\delta_{r}=\frac{r !(1+\alpha(r+1))}{\alpha+1} .
$$

From (2.5) we obtain the following corollary. 
Corollary 2.2. The mean deviation about mean of ALD is

$$
\delta_{1}=\frac{2 \alpha+1}{\alpha+1}
$$

Next we obtain various generating functions of the ALD through the following propositions.

Proposition 2.5. For any $t \in(-1,1)$, the moment generating function (m.g.f) of the ALD is

$$
M_{X}(t)=\frac{\left(1+\alpha-t^{2}+\alpha t^{2}\right)}{(\alpha+1)\left(1-t^{2}\right)^{2}} .
$$

Proof. By definition, we obtain the m.g.f of the ALD as follows, for any $t \in(-1,1)$

$$
\begin{aligned}
M_{X}(t) & =E\left(\mathrm{e}^{t X}\right)=\int_{-\infty}^{\infty} \mathrm{e}^{t x} \frac{1}{2(\alpha+1)}(1+\alpha|x|) \mathrm{e}^{-|x|} d x \\
& =\frac{1}{2(\alpha+1)}\left\{\int_{-\infty}^{0} \mathrm{e}^{(t+1) x}(1-\alpha x) d x+\int_{0}^{\infty} \mathrm{e}^{(t-1) x}(1+\alpha x) d x\right\} \\
& =\frac{1}{2(\alpha+1)}\left\{\frac{1}{1+t}+\frac{\alpha}{(1+t)^{2}}+\frac{1}{1-t}+\frac{\alpha}{(1-t)^{2}}\right\} \\
& =\frac{\left(1+\alpha-t^{2}+\alpha t^{2}\right)}{(\alpha+1)\left(1-t^{2}\right)^{2}} .
\end{aligned}
$$

Proposition 2.6. For any $t \in R$ and $i=\sqrt{-1}$, the characteristic function $\phi_{X}(t)$ of the ALD is given by

$$
\phi_{X}(t)=\frac{\left(1+\alpha+t^{2}-\alpha t^{2}\right)}{(\alpha+1)\left(1+t^{2}\right)^{2}} .
$$

Proposition 2.7. The $n^{t h}$ cumulant $\nu_{n}$ of the ALD is given by

$$
\nu_{n}=\left\{\begin{array}{cc}
\frac{2 !(3 \alpha+1)}{\alpha+1}, & n=2 \\
(n-1) !\left[\left\{2-\left(\frac{\sqrt{1-\alpha}}{\sqrt{(\alpha+1)}}\right)^{n}\right\}\left((-1)^{n}+1\right)\right], & n \geq 4 .
\end{array}\right.
$$

Proof. The cumulant generating function $\nu_{X}(t)$ of ALD is

$$
\begin{aligned}
\nu_{X}(t) & =\sum_{n=1}^{\infty} \nu_{n} \frac{t^{n}}{n !} \\
& =\log \left[M_{X}(t)\right]=\log \left[\frac{1+\alpha-t^{2}+\alpha t^{2}}{(\alpha+1)(1+t)^{2}(1-t)^{2}}\right] \\
& =\log \left(1+\alpha-t^{2}+\alpha t^{2}\right)-\log (1+\alpha)-2 \log (1+t)-2 \log (1-t) .
\end{aligned}
$$


Now, we expand the logarithmic terms in (2.10), to obtain the following.

$$
\begin{gathered}
\nu_{X}(t)=\frac{(3 \alpha+1) t^{2}}{\alpha+1}+\frac{\left(\alpha^{2}+6 \alpha+1\right) t^{4}}{2(\alpha+1)^{2}}+\frac{\left(3 \alpha^{3}+3 \alpha^{2}+9 \alpha+1\right) t^{6}}{3(\alpha+1)^{3}}+\ldots \\
+\frac{\left\{2-\left(\frac{\sqrt{1-\alpha}}{\sqrt{(\alpha+1)}}\right)^{n}\right\}\left((-1)^{n}+1\right) t^{n}}{n}+\ldots
\end{gathered}
$$

On equating coefficients of $\frac{t^{n}}{n !}$ in the right hand side expression of (2.9) and (2.11), we get the $n^{\text {th }}$ cumulant $\nu_{n}$ of the ALD is as given in (2.8). From (2.8), it can be noted that all the odd cumulants of the ALD are zero.

Remark 2.1. In particular, the $4^{\text {th }}, 6^{\text {th }}$ and $8^{\text {th }}$ cumulants of the ALD are respectively

$$
\begin{aligned}
\nu_{4} & =\frac{4 !\left(\alpha^{2}+6 \alpha+1\right)}{2(\alpha+1)^{2}} \\
\nu_{6} & =\frac{6 !\left(3 \alpha^{3}+3 \alpha^{2}+9 \alpha+1\right)}{3(\alpha+1)^{3}}
\end{aligned}
$$

and

$$
\nu_{8}=\frac{8 !\left(\alpha^{4}+12 \alpha^{3}+6 \alpha^{2}+12 \alpha+1\right)}{4(\alpha+1)^{4}} .
$$

Remark 2.2. Let $X \sim A L D(\alpha)$ with p.d.f. (2.1). Then $Y=\mu+\sigma X$ is said to have an extended alternative Laplace distribution (EALD) with parameters $\alpha, \mu$ and $\sigma$, which has the following p.d.f.

$$
f(y ; \alpha, \mu, \sigma)=\frac{1}{2 \sigma(\alpha+1)}\left(1+\alpha\left|\frac{y-\mu}{\sigma}\right|\right) \mathrm{e}^{-\left|\frac{y-\mu}{\sigma}\right|},
$$

for $-\infty<y<\infty, \alpha>0,-\infty<\mu<\infty$ and $\sigma>0$.

The cumulative distribution function of the EALD is given by

$$
F(y ; \alpha, \mu, \sigma)=\left\{\begin{array}{cc}
\frac{(\sigma+\alpha \mu+\alpha \sigma-\alpha y) \mathrm{e}^{\left(\frac{y-\mu}{\sigma}\right)}}{2(\alpha+1) \sigma}, & y<\mu \\
1-\frac{(\alpha y-\alpha \mu+\alpha \sigma+\sigma) \mathrm{e}^{-\left(\frac{y-\mu}{\sigma}\right)}}{2(\alpha+1) \sigma}, & y \geq \mu .
\end{array}\right.
$$

The further results of EALD are analogous to that of ALD as shown above and hence omitted.

\section{Reliability Measures}

This section deals with some reliability measures of ALD. Here we derive expressions for the reliability measures like survival function, hazard rate function and mean residual life function for the ALD so that they can be useful for studying reliability of a system involving one unit. First we present an expression for survival function of the distribution through the following proposition, its proof is omitted as it is straightforward. 
Proposition 3.1. The survival function $S(x)$ of $\operatorname{ALD}(\alpha)$ is given by

$$
\begin{aligned}
S(x) & =1-F(x ; \alpha) \\
& =\left\{\begin{array}{cl}
\frac{2(\alpha+1)-(1+\alpha-\alpha x) \mathrm{e}^{x}}{2(\alpha+1)}, & x<0 \\
\frac{(1+\alpha x+\alpha) \mathrm{e}^{-x}}{2(\alpha+1)}, & x \geq 0 .
\end{array}\right.
\end{aligned}
$$

Proposition 3.2. The failure rate function (or hazard rate function) of the ALD is given by

$$
h(x)=\left\{\begin{array}{cc}
\frac{(1-\alpha x) \mathrm{e}^{x}}{2(\alpha+1)-(1+\alpha-\alpha x) \mathrm{e}^{x}}, & x<0 \\
\frac{1+\alpha x}{1+\alpha+\alpha x}, & x \geq 0 .
\end{array}\right.
$$

The proof follows directly from the definition of failure rate function and hence omitted.

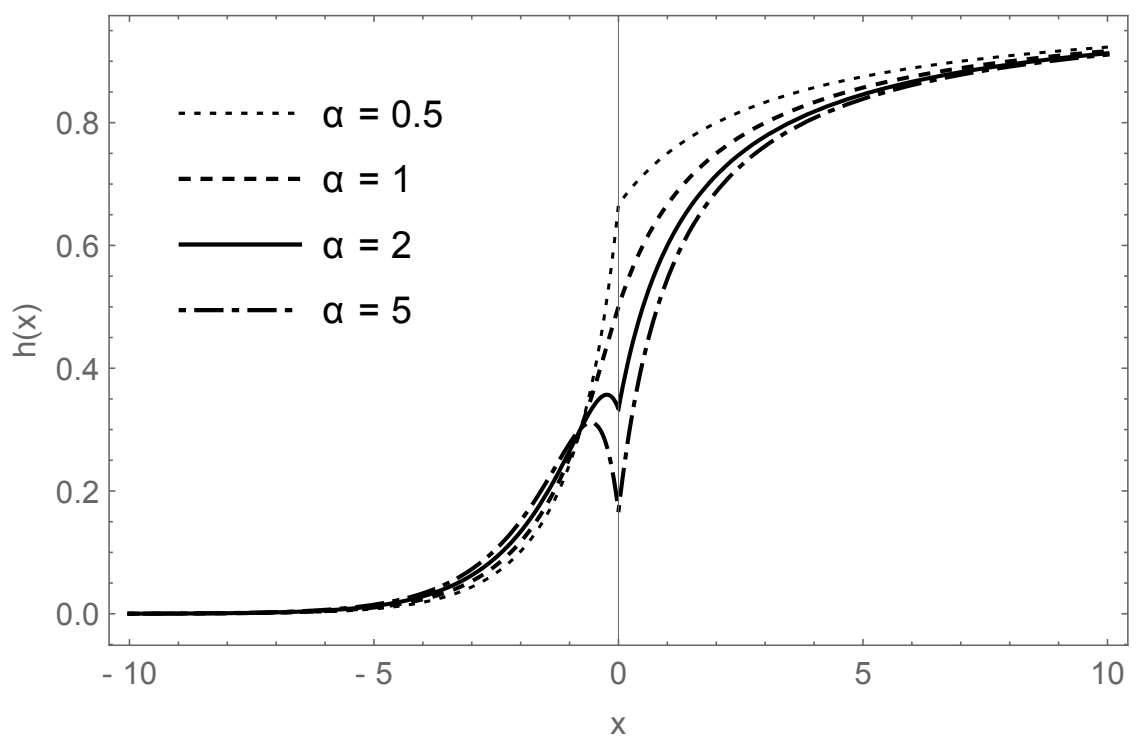

Figure 5: The hazard rate function of the ALD for different values of $\alpha$

It is to be noted that the monotonicity of failure rate function investigates whether the chance of occurrence of an event in the future over a fixed time interval increases or decreases over time. The failure rate function of the ALD is graphically represented in Figure 5. From Figure 5, we observe that the failure rate function of the ALD shows both increasing and decreasing nature.

Through the following theorem we precisely establishes the nature of the failure rate function of the ALD.

Theorem 3.1. If $X$ follows ALD with failure rate function as given in (3.2), then $X$ has decreasing failure rate (DFR) for any $\alpha>1$ such that $\frac{1-\alpha}{\alpha} \leq x<0$ and non-decreasing failure rate (IFR) elsewhere. 
Proof. On differentiating (3.2) with respect to $\mathrm{x}$, we have

$$
\frac{d}{d x} h(x)=\left\{\begin{array}{cc}
\frac{\mathrm{e}^{x}\left[e^{x} \alpha^{2}-2(\alpha+1)(\alpha-1+\alpha x)\right]}{\left[2(\alpha+1)-e^{x}(\alpha+1-\alpha x)\right]^{2}}, & x<0 \\
\frac{\alpha^{2}}{(\alpha+1+\alpha x)^{2}}, & x \geq 0 .
\end{array}\right.
$$

From the above expression, it follows that when $\alpha>1, \frac{d[h(x)]}{d x}<0$ for $\frac{1-\alpha}{\alpha}<x<0$ and $\frac{d[h(x)]}{d x}>0$, for all other values of $x$ and $\alpha$. Therefore, for $\alpha>1$ such that $\frac{1-\alpha}{\alpha} \leq x<0$, the failure rate function of ALD is DFR and when $\alpha \leq 1$ such that $x<\frac{1-\alpha}{\alpha}$, it is IFR. The failure rate function of the ALD is IFR for all values of $\alpha$ when $x \geq 0$.

In life testing situations, it is exciting to know about the expected additional lifetime of a component given that it has survived until a particular time. Through the following proposition we compute the mean residual life function of the ALD based on survival function given in (3.1).

Proposition 3.3. The mean residual life function (MRLF) of ALD is given by

$$
m(x)=\left\{\begin{array}{cc}
\frac{-2 x(\alpha+1)-(2 \alpha+1)+(2 \alpha+1-\alpha x) \mathrm{e}^{x}}{2(\alpha+1)-(1+\alpha-\alpha x) \mathrm{e}^{x}}, & x<0 \\
\frac{2 \alpha+1+\alpha x}{\alpha+1+\alpha x}, & x>0 .
\end{array}\right.
$$

Proof. For $x<0$, the MRLF of ALD is obtained by

$$
\begin{aligned}
m(x) & =E[X-x \mid X>x]=\frac{1}{1-F(x)} \int_{x}^{\infty}(1-F(t)) d t \\
& =\frac{1}{1-\frac{(1-\alpha x+\alpha) e^{x}}{2(\alpha+1)}} \int_{x}^{0}\left(1-\frac{(1-\alpha t+\alpha) \mathrm{e}^{t}}{2(\alpha+1)}\right) d t \\
& =\frac{-2 x(\alpha+1)-(2 \alpha+1)+e^{x}(2 \alpha+1-\alpha x)}{2(\alpha+1)-e^{x}(\alpha+1-\alpha x)}
\end{aligned}
$$

and for $x>0$, the MRLF of the ALD is given by

$$
\begin{aligned}
m(x) & =\frac{2(\alpha+1)}{(\alpha+1+\alpha x) \mathrm{e}^{-x}} \int_{x}^{\infty} \frac{(\alpha+1+\alpha t) \mathrm{e}^{-t}}{2(\alpha+1)} d t \\
& =\frac{2 \alpha+1+\alpha x}{\alpha+1+\alpha x} .
\end{aligned}
$$

\section{Order Statistics}

In this section, we discuss about the distribution of order statistics and the $q^{\text {th }}$ moment of the $k^{t h}$ order statistics of the ALD. 
Suppose $X_{1}, X_{2}, \ldots, X_{n}$ is a random sample from 2.1. Let $X_{1: n}<X_{2: n}<\ldots<X_{n: n}$ denote the corresponding order statistics. For $k \geq 1$, the p.d.f and c.d.f of the $k^{t h}$ order statistic $X_{k: n}$ of the ALD are given by

$$
f_{X_{k: n}}(x)=\left\{\begin{array}{cc}
\frac{n !(1-\alpha x) \mathrm{e}^{x}}{(k-1) !(n-k) ! 2(\alpha+1)} \sum_{r=0}^{n-k}\left(\begin{array}{c}
n-k \\
r
\end{array}\right)(-1)^{r}\left[\frac{[\alpha+1-\alpha x] \mathrm{e}^{x}}{2(\alpha+1)}\right]^{k+r-1}, & x<0 \\
\frac{n !(1+\alpha x) \mathrm{e}^{-x}}{(k-1) !(n-k) ! 2(\alpha+1)} \sum_{r=0}^{n-k}\left(\begin{array}{c}
n-k \\
r
\end{array}\right)(-1)^{r}\left[1-\frac{[\alpha+1+\alpha x] \mathrm{e}^{-x}}{2(\alpha+1)}\right]^{k+r-1}, & x \geq 0
\end{array}\right.
$$

and

$$
F_{X_{k: n}}(x)=\left\{\begin{array}{cl}
\sum_{j=k}^{n} \sum_{r=0}^{n-j}\left(\begin{array}{c}
n \\
j
\end{array}\right)\left(\begin{array}{c}
n-j \\
r
\end{array}\right)(-1)^{r}\left[\frac{[\alpha+1-\alpha x] \mathrm{e}^{x}}{2(\alpha+1)}\right]^{j+r}, & x<0 \\
\sum_{j=k}^{n} \sum_{r=0}^{n-j}\left(\begin{array}{c}
n \\
j
\end{array}\right)\left(\begin{array}{c}
n-j \\
r
\end{array}\right)(-1)^{r}\left[1-\frac{[\alpha+1+\alpha x] \mathrm{e}^{-x}}{2(\alpha+1)}\right]^{j+r}, & x \geq 0 .
\end{array}\right.
$$

Now, by using (4.1), we obtain the following proposition.

Proposition 4.1. For $q \geq 1$ and $1 \leq k \leq n$, the $q^{t h}$ moment of the $k^{t h}$ order statistic $X_{k: n}$ of the ALD is given by

$$
\begin{aligned}
E\left(X_{k: n}^{q}\right)= & \frac{n !}{(k-1) !(n-k) ! 2(\alpha+1)}\left\{\sum_{r=0}^{n-k} \sum_{m=0}^{k+r-1} \sum_{j=0}^{m}\left(\begin{array}{c}
n-k \\
r
\end{array}\right)\left(\begin{array}{c}
k+r-1 \\
m
\end{array}\right)\left(\begin{array}{c}
m \\
j
\end{array}\right)\right. \\
& \times \frac{(-1)^{r+q} \alpha^{k+r+j-m-1}(q+j) !}{[2(\alpha+1)]^{k+r-1}(k+r)^{q+j+2}}\left[\begin{array}{c}
k+r+\alpha(q+j+1)] \\
\end{array}\right. \\
& +\sum_{r=0}^{n-k} \sum_{m=0}^{k+r-1} \sum_{j=0}^{m} \sum_{i=0}^{j+1}\left(\begin{array}{c}
n-k \\
r
\end{array}\right)\left(\begin{array}{c}
k+r-1 \\
m
\end{array}\right)\left(\begin{array}{c}
m \\
j
\end{array}\right)\left(\begin{array}{c}
j+1 \\
i
\end{array}\right) \\
& \left.\times \frac{(-1)^{r+m} \alpha^{m+i-j}(q+i) !}{[2(\alpha+1)]^{m}(m+1)^{q+i+1}}\right\} .
\end{aligned}
$$


Proof. By definition,

$$
\begin{aligned}
& E\left(X_{k: n}^{q}\right)=\int_{-\infty}^{\infty} x^{q} f_{X_{k: n}}(x) d x \\
& =\frac{n !}{(k-1) !(n-k) ! 2(\alpha+1)}\left\{\sum_{r=0}^{n-k} \frac{\left(\begin{array}{c}
n-k \\
r
\end{array}\right)(-1)^{r} \alpha^{k+r-1}}{[2(\alpha+1)]^{k+r-1}}\right. \\
& \times \int_{-\infty}^{0}\left[x^{q}(1-\alpha x) \mathrm{e}^{(k+r) x}\left[1+\frac{1-\alpha x}{\alpha}\right]^{k+r-1}\right] d x \\
& \left.+\sum_{r=0}^{n-k}\left(\begin{array}{c}
n-k \\
r
\end{array}\right)(-1)^{r} \int_{0}^{\infty}\left[x^{q}(1+\alpha x) \mathrm{e}^{-x}\left[1-\frac{[\alpha+1+\alpha x] \mathrm{e}^{-x}}{2(\alpha+1)}\right]^{k+r-1}\right] d x\right\} \\
& =\frac{n !}{(k-1) !(n-k) ! 2(\alpha+1)}\left\{\sum_{r=0}^{n-k} \sum_{m=0}^{k+r-1} \sum_{j=0}^{m}\left(\begin{array}{c}
n-k \\
r
\end{array}\right)\left(\begin{array}{c}
k+r-1 \\
m
\end{array}\right)\left(\begin{array}{c}
m \\
j
\end{array}\right)\right. \\
& \times \frac{(-1)^{r+j} \alpha^{k+r+j-m-1}}{[2(\alpha+1)]^{k+r-1}} \int_{-\infty}^{0}\left[x^{q+j}(1-\alpha x) \mathrm{e}^{(k+r) x}\right] d x \\
& +\sum_{r=0}^{n-k} \sum_{m=0}^{k+r-1} \sum_{j=0}^{m}\left(\begin{array}{c}
n-k \\
r
\end{array}\right)\left(\begin{array}{c}
k+r-1 \\
m
\end{array}\right)\left(\begin{array}{c}
m \\
j
\end{array}\right) \frac{(-1)^{r+m} \alpha^{m-j}}{[2(\alpha+1)]^{m}} \\
& \left.\times \int_{0}^{\infty}\left[x^{q} \mathrm{e}^{-(m+1) x} \sum_{i=0}^{j+1}\left(\begin{array}{c}
j+1 \\
i
\end{array}\right) \alpha^{i} x^{i}\right] d x\right\},
\end{aligned}
$$

by binomial expansion. Now, on evaluating the integrals, we get (4.3).

\section{Entropy}

In this section we obtain an entropy measure of the ALD. An entropy of a random variable $X$ is a measure of variation of the uncertainty. A popular entropy measure introduced by Rényi (1961) is Rényi entropy. Through the following proposition we obtain an expression for the Rényi entropy of the ALD.

Proposition 5.1. The Rényi entropy $I_{R}(\gamma)$ of the ALD is obtained as

$$
\begin{array}{r}
I_{R}(\gamma)=\frac{1}{1-\gamma}\{-(\gamma-1) \log (2)-\gamma \log (\alpha+1)-\log (\gamma) \\
\left.+\log \left({ }_{2} F_{1}\left(-\gamma, 1 ; ; \frac{-\alpha}{\gamma}\right)\right)\right\},
\end{array}
$$

where

$$
{ }_{2} F_{1}(a, b ; c ; \theta)=\sum_{j=0}^{\infty} \frac{(a)_{j}(b)_{j}}{(c)_{j}} \frac{\theta^{j}}{j !}
$$

is the Gauss Hypergeometric Function. 
Proof. By definition, Rényi entropy of a distribution with p.d.f $f($.$) is given by$

$$
I_{R}(\gamma)=\frac{1}{1-\gamma} \log \left\{\int f^{\gamma}(x) d x\right\}
$$

where $1 \neq \gamma>0$. Thus, for the ALD with p.d.f (2.1), note that

$$
\begin{aligned}
\int f^{\gamma}(x) d x & =\int_{-\infty}^{\infty}\left[\frac{1}{2(\alpha+1)}(1+\alpha|x|) e^{-|x|}\right]^{\gamma} d x \\
& =\frac{1}{2^{\gamma}(\alpha+1)^{\gamma}}\left\{\int_{-\infty}^{0}(1-\alpha x)^{\gamma} e^{\gamma x} d x+\int_{0}^{\infty}(1+\alpha x)^{\gamma} e^{-\gamma x} d x\right\} .
\end{aligned}
$$

Now, using the series expansions,

$$
(1+x)^{a}=\sum_{j=0}^{\infty}(a+1-j)_{j} \frac{x^{j}}{j !}
$$

and

$$
(1-x)^{a}=\sum_{j=0}^{\infty}(a+1-j)_{j} \frac{(-1)^{j} x^{j}}{j !},
$$

for any $a \in R$ and $(x)_{k}=x(x+1) \ldots(x+k-1)$ for $k \geq 1$ with $(x)_{0}=1$. Then, we get

$$
\begin{aligned}
\int f^{\gamma}(x) d x=\frac{1}{2^{\gamma}(\alpha+1)^{\gamma}}\left\{\int_{-\infty}^{0} \sum_{j=0}^{\infty}(\gamma+1-j)_{j} \frac{(-1)^{j}(\alpha x)^{j}}{j !} e^{\gamma x} d x\right. \\
\left.\quad+\int_{0}^{\infty} \sum_{j=0}^{\infty}(\gamma+1-j)_{j} \frac{(\alpha x)^{j}}{j !} e^{-\gamma x} d x\right\} \\
=\frac{1}{2^{\gamma-1}(\alpha+1)^{\gamma}} \sum_{j=0}^{\infty}(\gamma+1-j)_{j} \frac{\alpha^{j} \Gamma(j+1)}{j !(\gamma)^{j+1}} .
\end{aligned}
$$

Now, by applying the identity $(a-n)_{n}=(-1)^{n}(1-a)_{n}$ in (5.6), we get

$$
\int f^{\gamma}(x) d x=\frac{1}{2^{\gamma-1} \gamma(\alpha+1)^{\gamma}}{ }^{2} F_{1}\left(-\gamma, 1 ; ; \frac{-\alpha}{\gamma}\right),
$$

which implies (5.1).

\section{Estimation}

\subsection{Parameter Estimation of ALD}

Let $X_{1}, X_{2}, \ldots, X_{n}$ be a random sample of size $n$ from ALD with p.d.f given in (2.1). Let $X_{(1)}, X_{(2)}, \ldots, X_{(n)}$ be the ordered sample. Assume $X_{(r)}<0<X_{(r+1)}$, for a particular $r=$ 
$1,2, \ldots, n$. Then,the loglikelihood function $l(\alpha)$ of the sample is the following, in which $\sum_{J_{i}}$, denotes the summation over the set $J_{i}$ such that $J_{1}=\left\{j: Y_{(j)}<0\right.$, for $\left.j=1,2, \ldots, r\right\}$ and $J_{2}=\left\{j: Y_{(j)} \geq 0\right.$, for $\left.j=r+1, \ldots, n\right\}$.

$$
l(\alpha)=-n \ln (2)-n \ln (\alpha+1)+\sum_{J_{1}} x_{i}-\sum_{J_{2}} x_{i}+\sum_{J_{1}} \ln \left(1-\alpha x_{i}\right)+\sum_{J_{2}} \ln \left(1+\alpha x_{i}\right) .
$$

On differentiating (6.1) with respect to the parameter $\alpha$ and then equating to zero, we obtain the following likelihood equation.

$$
\frac{-n}{\alpha+1}-\sum_{J_{1}} \frac{x_{i}}{\left(1-\alpha x_{i}\right)}+\sum_{J_{2}} \frac{x_{i}}{\left(1+\alpha x_{i}\right)}=0
$$

On solving the equation (6.2), we get the maximum likelihood estimator of the parameter $\alpha$ of the ALD.

\subsection{Estimation of Parameters of EALD}

Let $Y_{1}, Y_{2}, \ldots, Y_{n}$ be a random sample of size $n$ from EALD with p.d.f given in (2.12). Let $Y_{(1)}, Y_{(2)}, \ldots, Y_{(n)}$ be the ordered sample. Assume $Y_{(r)}<\mu<Y_{(r+1)}$, for a particular $r=$ $1,2, \ldots, n$. Then,the loglikelihood function $l(\underline{\alpha})=l(\alpha, \mu, \sigma)$ of the sample is the following, in which $\sum_{I_{j}}$, denotes the summation over the set $I_{j}$ such that $I_{1}=\left\{i: Y_{(i)}<\mu\right.$, for $\left.i=1,2, \ldots, r\right\}$ and $I_{2}=\left\{i: Y_{(i)} \geq \mu\right.$, for $\left.i=r+1, \ldots, n\right\}$.

$$
\begin{aligned}
l(\underline{\alpha})=-n \ln (2) & -n \ln (\sigma)-n \ln (\alpha+1)-\frac{1}{\sigma} \sum_{I_{1}}\left(\mu-y_{i}\right)-\frac{1}{\sigma} \sum_{I_{2}}\left(y_{i}-\mu\right) \\
+ & \sum_{I_{1}} \ln \left(1+\alpha\left(\frac{\mu-y_{i}}{\sigma}\right)\right)+\sum_{I_{2}} \ln \left(1+\alpha\left(\frac{y_{i}-\mu}{\sigma}\right)\right) .
\end{aligned}
$$

On differentiating (6.3) with respect to the parameters $\alpha, \mu$ and $\sigma$ and then equating to zero, we obtain the following likelihood equations.

$$
\begin{aligned}
& \frac{-n}{\alpha+1}+\sum_{I_{1}} \frac{\left(\mu-y_{i}\right)}{\sigma\left[1+\alpha\left(\frac{\mu-y_{i}}{\sigma}\right)\right]}+\sum_{I_{2}} \frac{\left(y_{i}-\mu\right)}{\sigma\left[1+\alpha\left(\frac{y_{i}-\mu}{\sigma}\right)\right]}=0 \\
& \frac{n-2 r}{\sigma}+\frac{\alpha}{\sigma} \sum_{I_{1}} \frac{1}{1+\alpha\left(\frac{\mu-y_{i}}{\sigma}\right)}-\frac{\alpha}{\sigma} \sum_{I_{2}} \frac{1}{1+\alpha\left(\frac{y_{i}-\mu}{\sigma}\right)}=0
\end{aligned}
$$

and

$$
\begin{array}{r}
-\frac{n}{\sigma}+\frac{1}{\sigma^{2}} \sum_{I_{1}}\left(\mu-y_{i}\right)+\frac{1}{\sigma^{2}} \sum_{I_{2}}\left(y_{i}-\mu\right)-\frac{\alpha}{\sigma^{2}} \sum_{I_{1}} \frac{\left(\mu-y_{i}\right)}{1+\alpha\left(\frac{\mu-y_{i}}{\sigma}\right)} \\
-\frac{\alpha}{\sigma^{2}} \sum_{I_{2}} \frac{\left(y_{i}-\mu\right)}{1+\alpha\left(\frac{y_{i}-\mu}{\sigma}\right)}=0 .
\end{array}
$$


On solving the equations (6.4) - (6.6) we get the maximum likelihood estimators of the parameters $\alpha, \mu$ and $\sigma$ of the EALD.

\section{Applications}

For numerical illustration, in this section we consider the following three different types of data sets. In Data set 1, we consider a data on market shares of airlines of 1000 U.S. domestic routes for $4^{\text {th }}$ quarter of 2002, which one may find from the website of U.S. department of transportation: https://www.transportation.gov.

The data set 2 is taken from Weisberg (2005), which discusses the variation in rent paid in 1977 for agricultural land planted to alfalfa of the 67 countries in Minnesota. Alfalfa is a high protein crop that is suitable feed for dairy cows. In this data set our variable under study is the average rent paid for all tillable land.

Data set 2 : 15.5, 22.29, 12.36, 31.84, 83.9, 72.25, 27.14, 40.41, 12.42, 69.42, 48.46, 69., 26.09, $62.83,77.06,58.83,59.48,8.5,20.64,81.4,18.92,50.32,21.33,46.85,65.94,38.68,51.19,59.42$, 24.64, 26.94, 46.2, 26.86, 20., 62.52, 56., 71.41, 65., 36.28, 59.88, 23.62, 24.2, 17.09, 44.56, 34.46, $31.55,26.94,58.71,65.74,69.05,57.54,21.73,5 ., 51 ., 18.25,69.88,26.68,75.73,41.77,48.5$, $21.89,38.33,53.95,17.17,82 ., 40.6,53.89,54.17$

The dataset 3 is a voltage drop data which describes the battery voltage drop in a guided missile motor observed over the time of missile flight. This dataset is taken from Montgomery et al. (2015). Data set $3: 8.33,8.23,7.17,7.14,7.31,7.6,7.94,8.3,8.76,8.71,9.71,10.26,10.91,11.67,11.76$, $12.81,13.3,13.88,14.59,14.05,14.48,14.92,14.37,14.63,15.18,14.51,14.34,13.81,13.79$, $13.05,13.04,12.6,12.05,11.15,11.15,10.14,10.08,9.78,9.8,9.95,9.51$

By using MATHEMATICA software, we have fitted the models EALD $(\alpha, \mu, \sigma)$ and Laplace $(\mu, \sigma)$ to all the above three data sets by utilizing maximum likelihood estimation procedure. The Kolmogorov-Smirnov statistic (KSS) is obtained in each case inorder to assess the goodness of fit. The numerical results obtained are presented in Tables 1,2 and 3. For model comparison, we have also computed some well known information criterion-such as Akaike's Information Criterion (AIC), the Bayesian Information Criterion (BIC) and the corrected Akaike's Information Criterion (AICc), which are also included in Tables 1, 2 and 3.

Based on AIC values one may also compute probability of betterment to select a suitable model for a data in hand. Probability of betterment $\left(p_{i}\right)$ is the relative probability to $i^{\text {th }}$ model over the model with minimum AIC and it is obtained using the formulae (see Burnham and Anderson (2004) for more details)

$$
p_{i}=\exp \left\{\frac{-\Delta_{i}}{2}\right\},
$$

where $\Delta_{i}=$ AIC of $i^{t h}$ model - AIC of model having minimum value. Here, the probability of betterment has been computed for Laplace distribution over EALD and hence we denote it as $p_{2}$. For data set $1, p_{2} \approx 0$, which indicates that there is approximately $0 \%$ probability that Laplace distribution will be a better model compared to EALD for analyzing the data. Similarly, for the data 
Table 1: Estimated values of the parameters for the EALD and Laplace distribution with respective KSS, AIC, BIC and AICc values for the Data set 1.

\begin{tabular}{ccc} 
Estimates of the Parameters & Laplace $(\mu, \sigma)$ & $\operatorname{EALD}(\alpha, \mu, \sigma)$ \\
\hline$\hat{\alpha}$ & - & 3.0574 \\
$\hat{\mu}$ & 59.24 & 59.2912 \\
$\hat{\sigma}$ & 15.8857 & 9.05925 \\
\hline KSS & 0.06341 & 0.03319 \\
AIC & 8921.14 & 8815.08 \\
BIC & 8930.95 & 8829.8 \\
AICc & 8921.15 & 8815.1 \\
\hline
\end{tabular}

Table 2: Estimated values of the parameters for the EALD and Laplace distribution with respective KSS, AIC, BIC and AICc values for the Data set 2.

\begin{tabular}{ccc} 
Estimates of the Parameters & Laplace $(\mu, \sigma)$ & $\operatorname{EALD}(\alpha, \mu, \sigma)$ \\
\hline$\hat{\alpha}$ & - & 60868.8 \\
$\hat{\mu}$ & 44.56 & 43.2383 \\
$\hat{\sigma}$ & 18.4687 & 9.24426 \\
\hline KSS & 0.16352 & 0.11797 \\
AIC & 621.636 & 602.425 \\
BIC & 626.045 & 609.039 \\
AICc & 621.823 & 602.806 \\
\hline
\end{tabular}

Table 3: Estimated values of the parameters for the EALD and Laplace distribution with respective KSS, AIC, BIC and AICc values for the Data set 3.

\begin{tabular}{ccc} 
Estimates of the Parameters & Laplace $(\mu, \sigma)$ & $\operatorname{EALD}(\alpha, \mu, \sigma)$ \\
\hline$\hat{\alpha}$ & - & 61458.2 \\
$\hat{\mu}$ & 11.15 & 11.4328 \\
$\hat{\sigma}$ & 2.24965 & 1.12602 \\
\hline KSS & 0.152183 & 0.102028 \\
AIC & 209.157 & 197.642 \\
BIC & 212.584 & 202.782 \\
AICc & 209.472 & 198.29 \\
\hline
\end{tabular}


An alternative Laplace distribution

Table 4: Estimates of the parameters and the corresponding bias and mean square error

\begin{tabular}{ccccc}
\hline Sample Size & Parameters & Estimates & M.S.E & Bias \\
\hline \multirow{2}{*}{$\mathrm{n}=200$} & $\alpha$ & 4.7339 & 4.9213 & 0.2339 \\
& $\mu$ & 4.9536 & 0.0881 & -0.0464 \\
& $\sigma$ & 2.0426 & 0.1371 & 0.0426 \\
\hline \multirow{2}{*}{$\mathrm{n}=350$} & $\alpha$ & 4.7027 & 1.8526 & 0.2027 \\
& $\mu$ & 5.0198 & 0.0318 & 0.0198 \\
& $\sigma$ & 2.0367 & 0.1064 & 0.0367 \\
\hline \multirow{2}{*}{$\mathrm{n}=500$} & $\alpha$ & 4.3579 & 1.3140 & -0.1421 \\
& $\mu$ & 5.0084 & 0.0238 & 0.0084 \\
& $\sigma$ & 2.0190 & 0.0060 & 0.0190 \\
\hline
\end{tabular}

set 2, $p_{2}=0.000067$, which indicates that there is $0.0067 \%$ probability that Laplace distribution will be a better model compared to EALD for analyzing data set 2. Also we can observe that for the data set $3, p_{2}=0.003159$, which indicates that there is $0.316 \%$ probability that Laplace distribution will be a better model compared to EALD for analyzing data set 3. It is clear from the tables and also from the evaluated values of probability of betterment that the EALD is a more appropriate model to the data sets when compared to Laplace distribution.

\section{Simulation}

In this section, we investigate the behaviour of the maximum likelihood estimators for a finite sample size $n$ by conducting a brief simulation study as follows. We have generated samples of sizes 200 , 350 and 500 from the EALD using inverse c.d.f transformation. From the generated samples, we obtain maximum likelihood estimates of $\alpha, \mu$ and $\sigma$. Then we compute average bias and average mean square error (MSE) occurred in the estimates of $\alpha, \mu$ and $\sigma$ from 1000 Monte Carlo replicates. The computation of average bias and MSE are based on the following formulas,

$$
\begin{aligned}
\text { Bias } & =\frac{1}{1000} \sum_{i=1}^{1000}\left(\hat{h_{i}}-h_{i}\right) \\
M S E & =\frac{1}{1000} \sum_{i=1}^{1000}\left(\hat{h_{i}}-h_{i}\right)^{2}
\end{aligned}
$$

where, $\hat{h_{i}}$ and $h_{i}$ are the estimated and true values of either $\alpha$ or $\mu$ or $\sigma$. The true values of the parameters are $\alpha=4.5, \mu=5$ and $\sigma=2$. The results obtained are presented in Table 4 . 
From Table 4, it can be observed that both the bias and MSE decreases as the sample size increases. Thus, the simulation study supports the appropriateness of our model and accuracy on the estimates obtained.

\section{Conclusion}

An alternative to the well-known Laplace distribution has been proposed through the name "alternative Laplace distribution (ALD)" in the present study. The ALD possess both bimodal and unimodal nature whereas the Laplace distribution is always unimodal. While the measure of peakedness for Laplace distribution is a constant, in case of the ALD, the kurtosis is a function of the parameter allowing wider range. The heavier tails of ALD when compared to Laplace distribution enables the slower death of the model. The failure rate of ALD exhibits both increasing and decreasing behaviour over the real line, whereas the failure rate of Laplace distribution is increasing in the negative support and remains constant in the non-negative support of the random variable. These characterizations of the ALD makes it more suitable in practical situations as an alternative to Laplace distribution. The feasibility of the location-scale extended class of ALD over the Laplace distribution in modelling real life data sets especially in industrial and financial sectors have been illustrated in the present study.

\section{Acknowledgements}

The authors wish to express their sincere gratitude to the Editor in Chief and anonymous referees for their valuable comments on an earlier version of the paper that improved the quality of the manuscript to a greater extent.

\section{References}

Burnham, K. P. and Anderson, D. R. (2004), "Multimodel inference: understanding AIC and BIC in model selection," Sociological methods \& research, 33, 261-304.

Cordeiro, G. M. and Lemonte, A. J. (2011), “The beta Laplace distribution,” Statistics \& Probability Letters, 81, 973-982.

Jose, K. K. and Thomas, M. M. (2014), "Multivariate normal-Laplace distribution and processes," Statistica, 74, 27-44.

Kozubowski, T. J., Podgórski, K., et al. (2016), "Esscher-transformed Laplace distribution revisited," Brazilian Journal of Probability and Statistics, 30, 432-434.

Li, L. (2017), "Bayes Estimation of Parameter of Laplace Distribution Under a New LINEX-Based Loss Function," International Journal of Data Science and Analysis, 3, 85-89. 
Liu, Y. and Kozubowski, T. J. (2015), “A folded Laplace distribution,” Journal of Statistical Distributions and Applications, 2, 10-26.

Mahmoudvand, R., Faradmal, J., Abbasi, N., and Lurz, K. (2015), "A New Modification in the Classical Laplace Distribution,” Journal of The Iranian Statistical Society, 14, 93-118.

Montgomery, D. C., Peck, E. A., and Vining, G. G. (2015), Introduction to Linear Regression Analysis, John Wiley \& Sons, New Jersey.

Nassar, M. M. (2016), “The Kumaraswamy Laplace Distribution,” Pakistan Journal of Statistics and Operation Research, 12, 609-624.

Rényi, A. (1961), "On measures of entropy and information," in Fourth Berkeley Symposium on Mathematical Statistics and Probability, 1, 547-561.

Weisberg, S. (2005), Applied Linear Regression, John Wiley \& Sons, New York.

Received: August 19, 2019

Accepted: October 15, 2019 